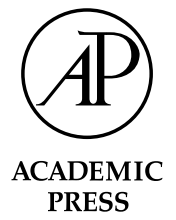

\title{
Peroxisomal membrane monocarboxylate transporters: evidence for a redox shuttle system?
}

\author{
Grant B. McClelland, ${ }^{1}$ Savita Khanna, ${ }^{2}$ Gilda F. González, ${ }^{3}$ \\ C. Eric Butz, and George A. Brooks* \\ Exercise Physiology Laboratory, Department of Integrative Biology, University of California, Berkeley, CA 94720-3140, USA
}

Received 21 February 2003

\begin{abstract}
One of the many functions of liver peroxisomes is the $\beta$-oxidation of long-chain fatty acids. It is essential for the continuation of peroxisomal $\beta$-oxidation that a redox shuttle system exist across the peroxisomal membrane to reoxidize NADH. We propose that this redox shuttle system consists of a substrate cycle between lactate and pyruvate. Here we present evidence that purified peroxisomal membranes contain both monocarboxylate transporter 1 (MCT 1) and MCT 2 and that along with peroxisomal lactate dehydrogenase $(\mathrm{pLDH})$ form a Peroxisomal Lactate Shuttle. Peroxisomal $\beta$-oxidation was greatly stimulated by the addition of pyruvate and this increase was partially inhibited by the addition of the MCT blocker $\alpha$-cyano-4-hydroxycinnamate (CINN). We also found that peroxisomes generated lactate in the presence of pyruvate. Together these data provide compelling that the Peroxisome Lactate Shuttle helps maintain organelle redox and the proper functioning of peroxisomal $\beta$-oxidation.

(C) 2003 Elsevier Science (USA). All rights reserved.
\end{abstract}

Keywords: Peroxisome; Monocarboxylate transporter 1; Monocarboxylate transporter 2; Lactate shuttle; Lactate dehydrogenase; $\beta$-Oxidation; Liver; Reduction-oxidation; Pyruvate; Catalase

Liver peroxisomes are cellular organelles important not only for the detoxification process but also for the $\beta$ oxidation of long-chain fatty acids [1]. It is essential for the continued functioning of peroxisomes that proper intra-organelle reduction-oxidation (redox) balance is maintained. This ensures the reoxidation of NADH produced by $\beta$-oxidation and the continued breakdown of fatty acids. Redox balance in many organelles is maintained in part by substrate shuttles. This would entail a shuttle system in peroxisomes that neither generates products nor consumes any substrates. In other organelles, such shuttles often involve a dehydrogenase.

\footnotetext{
${ }^{*}$ Corresponding author. Fax: 1-510-643-2439.

E-mail address: gbrooks@socrates.Berkeley.EDU (G.A. Brooks).

${ }^{1}$ Present address: Department of Biology, Queen's University, Kingston, Canada.

${ }^{2}$ Present address: Dorothy M. Davis Heart and Lung Research Institute, Ohio State University, Columbus, $\mathrm{OH}$, USA.

${ }^{3}$ Present address: Ernest Gallo Clinic and Research Center, Emeryville, CA, USA.
}

Peroxisomal membranes in yeast have been found to be impermeable to $\mathrm{NAD}^{+} / \mathrm{NADH}$ (Saccharomyces cerevisiae) [2] and so a shuttle system appears mandatory. Moreover, the recent discovery of a peroxisomal lactate dehydrogenase (L-lactate:NAD ${ }^{+}$oxidoreductase (pLDH); EC 1.1.1.27) [3], plus the absence of any other glycolytic enzymes in this organelle, points to this dehydrogenase as a site of NADH reoxidation. Indeed, the addition of pyruvate to purified peroxisomes has been found to increase the rate of $\beta$-oxidation of palmitoyl-CoA [4]. As well, the LDH inhibitor, oxamate, decreased the reoxidation of NADH in isolated peroxisomes and treatment of rats with the hypolipidemic drug bezafibrate causes parallel increases in both LDH and $\beta$-oxidation enzymes [3]. To play their role in peroxisomal redox balance, pyruvate and lactate must be transported across the peroxisome membrane.

At physiological $\mathrm{pH}$ pyruvate and lactate exist almost entirely in anionic forms and in this charged state cannot pass through cellular membranes by simple diffusion [5]. Recently, it has been found that the 
transport of lactate and pyruvate in and out of cells [6] and into mitochondria [7], is facilitated by protonlinked monocarboxylate transporters (MCTs). Lactate to pyruvate exchange has been proposed to function as a redox shuttle in mitochondria of skeletal muscles and liver, aided by an intramitochondrial pool of $\mathrm{LDH}$ $[7,8]$. The same sort of shuttle system has been proposed for other systems because LDH has also been located in the mitochondria of sperm from a variety of species $[9,10]$. MCTs have tissue and organelle-specific distribution with MCT 2 being the predominant isoform in liver [6]. Establishing the presence of MCT 2 in peroxisomal membranes and demonstrating a linkage between pyruvate-lactate exchange and $\beta$-oxidation would be compelling evidence for a redox shuttle between this organelle and the cytosol. The absence of glycolysis in this organelle strongly suggests that this shuttle serves to maintain redox balance (as outlined in Fig. 1) and not glycolysis or some other metabolic function.

Evidence for such a Peroxisome Lactate Shuttle would consist of the detection of the necessary metabolic machinery and demonstration of its functionality in intact organelles. To address this issue we isolated highly purified peroxisomes from rat livers to look for the presence of MCTs and pLDH. We also measured lactate release and $\beta$-oxidation in intact peroxisomes in the presence of inhibitors of pyruvate uptake and $\mathrm{LDH}$ activity. We hypothesize that MCTs are present on the peroxisomal membrane and that along with $\mathrm{pLDH}$ form a redox shuttle. This shuttle aids in the reoxidizing of $\mathrm{NADH}$ in the peroxisome and allows $\beta$-oxidation to continue.

\section{Materials and methods}

Materials. Optiprep (a sterile 60\% (w/v) Iodixanol solution) was obtained from Nycomed. Sucrose, EDTA, Mops, PMSF, DTT, and $\varepsilon-$ aminocaproic were purchased from Sigma. $\left[1-{ }^{14} \mathrm{C}\right]$ palmitoyl-CoA was obtained from NEN.

Animals. All protocols were approved by the University of California, Berkeley Animal Care and Use Committee. Male and female Sprague-Dawley rats $(200-300 \mathrm{~g})$ were fed standard rat chow and housed under standard light and temperature conditions. Animals were euthanized via Nembutal injection $(120 \mathrm{mg} / \mathrm{kg} \mathrm{IP})$. Liver samples were taken for peroxisome purification.

Purification of peroxisomes. Peroxisomes were purified as described previously [11]. Briefly, livers were finely minced with scissors in icecold homogenization buffer $(0.25 \mathrm{M}$ sucrose, $1 \mathrm{mM}$ EDTA, $0.1 \%(\mathrm{v} / \mathrm{v})$ ethanol, $10 \mathrm{mM}$ Mops- $\mathrm{NaOH}$, pH 7.4, with $0.2 \mathrm{mM}$ PMSF, $0.2 \mathrm{mM}$ DTT, and $1 \mathrm{mM}$-aminocaproic acid) and transferred to a PotterElvehjem homogenizer (Teflon on glass) and homogenized. To pellet the nuclei and heavy mitochondria, liver homogenates were centrifuged at $3000 \mathrm{~g}$ in a fixed-angle rotor for $10 \mathrm{~min}$ at $4{ }^{\circ} \mathrm{C}$. To produce a light mitochondrial pellet the supernatant (S) was centrifuged at $17,000 \mathrm{~g}$ for $10-15 \mathrm{~min}$. This pellet (LM) was resuspended in homogenization buffer by using loose-fitting Dounce homogenizer (2-3 strokes). The volume was adjusted to approximately $15 \mathrm{ml} / 10 \mathrm{~g}$ tissue and an equal volume of gradient solution (5 vol. of $60 \%$ Iodixanol) was mixed with 1 vol. of dilution medium $(0.25 \mathrm{M}$ sucrose, $6 \mathrm{mM}$ EDTA, $0.6 \%$ (v/v) ethanol, $60 \mathrm{mM}$ Mops- $\mathrm{NaOH}, \mathrm{pH} 7.4$, with $0.2 \mathrm{mM}$ PMSF, $0.2 \mathrm{mM}$ DTT, and $1 \mathrm{mM}$-aminocaproic acid). This homogenate was centrifuged at $180,000 \mathrm{~g}$ for $2.5 \mathrm{~h}$ at $4{ }^{\circ} \mathrm{C}$ in a fixedangle rotor (Beckman Type NVT 65) using slow acceleration and deceleration modes. After centrifugation, fractions were removed starting from the bottom with needle and syringe. These fractions were highly purified peroxisomes $(\mathrm{P})$, peroxisomal $(\mathrm{P}+\mathrm{M})$, middle layer (MID), mitochondrial-rich (M), and lysosomal (T) fractions. For lactate release and $\beta$-oxidation measurements equal amounts of homogenization buffer were added to $\mathrm{P}$ and $\mathrm{P}+\mathrm{M}$ layers and centrifuged at $30,000 \mathrm{~g}$ for $10 \mathrm{~min}$ at $4{ }^{\circ} \mathrm{C}$. The pellet was resuspended in homogenization buffer for lactate release and $\beta$-oxidation or a buffer containing $10 \mathrm{mM}$ Tris and $1 \mathrm{mM}$ EDTA, pH 7.4, for Western blots.

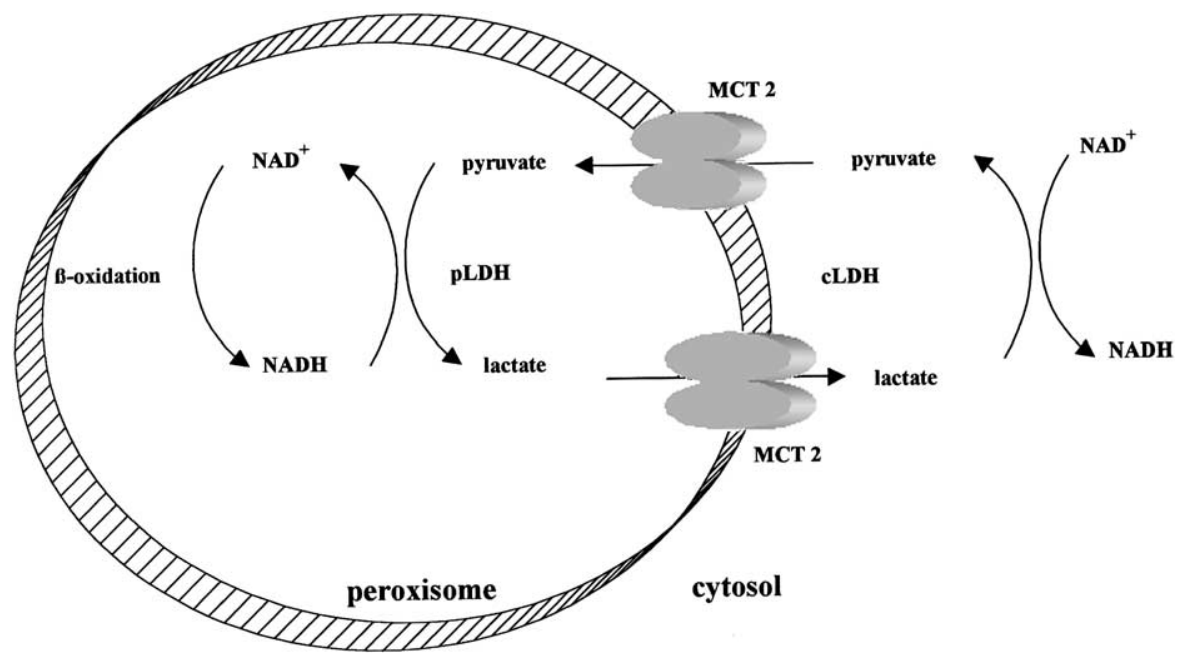

Fig. 1. A schematic showing the putative lactate-pyruvate redox shuttle system for the re-oxidation of NADH generated by $\beta$-oxidation in peroxisomes. Cytosolic pyruvate is transported into the peroxisome via monocarboxylate transporters 2 (MCT 2). Once in the peroxisome it is converted to lactate by a peroxisomal LDH (pLDH). The resulting reoxidation of NADH to NAD allows $\beta$-oxidation to continue. The resulting lactate can be converted back to pyruvate in the cytosol by cLDH. 
LDH separation and analysis-electrophoresis. LDH isoenzymes present in different fractions of the liver homogenate were separated by adding $6 \mu \mathrm{g}$ protein to agarose (1\%) gels (Reliant precast gels; FMC) and electrophoresing at $90 \mathrm{~V}$ for $30 \mathrm{~min}$ by using a Bio-Rad Sub-Cell system. An electrophoretic marker (LDH Isotrol; Sigma) containing LDH isoenzymes $1-5$ was used in some gels as an aid in identification of isoenzymes. After electrophoresis, the areas of isoenzyme activity were visualized by using a colorimetric procedure (Sigma Procedure 705). The gels were fixed in 5\% acetic acid. LDH isoenzyme bands were scanned and quantified by using a Bio-Rad GS-700 imaging densitometer.

Immunoblots. The presence of moncarboxylate transporters in purified peroxisomal membranes was detected by standard Western blot techniques. Affinity-purified polyclonal antibody for MCT 1 was produced by immunizing rabbits with synthetic peptide as described elsewhere [12]. MCT 2 antibody was chicken polyclonal specific to rat (Chemicon, International). Catalase content was detected with a rabbit-specific antibody (Rockland) and mitochondrial contamination of purified peroxisomal fractions was assayed with anti-bovine NADHubiquinol oxidoreductase, mouse monoclonal (Complex I) (Molecular Probes). Protein content of each sample was determined by the bicinchoninic acid assay (Pierce) using BSA as a standard. Equal amounts of protein were separated on $10 \%$ SDS-PAGE and transferred by electroblotting on polyvinylidene difluoride (PVDF) membranes (Invitrogen). Membranes were incubated for $1 \mathrm{~h}$ at room temperature or overnight at $4{ }^{\circ} \mathrm{C}$ in $10 \%$ blocking buffer $(150 \mathrm{mM}$ $\mathrm{NaCl}, 0.1 \%$ Tween 20 , and $50 \mathrm{mM}$ Tris, pH 7.5 (TTBS), and $10 \%$ nonfat dried milk) before incubation with antibodies for $2 \mathrm{~h}$ at room temperature or overnight at $4{ }^{\circ} \mathrm{C}$ in $5 \%$ blocking buffer (in TBS). Membranes were washed once for $15 \mathrm{~min}$ and then twice for $5 \mathrm{~min}$ in TTBS followed by a 10-min wash in TBS. They were then incubated for $90 \mathrm{~min}$ at room temperature with secondary antibody in $5 \%$ blocking buffer in TBS. Membranes were washed as above and protein expression was then detected by enhanced chemiluminescence (ECL) by standard methods (Renaissence; NEN). Autoradiographic films (Kodak X-Omat Blue XB-1) were exposed to membranes and developed (Konica QX-130A Plus). Band densities were determined with a Bio-Rad GS-700 densitometer and quantified with Molecular Analyst software (Bio-Rad Laboratories). Molecular weight standards were used to identify appropriate antibody binding (Rainbow Marker, Amersham Life Sciences). The membranes were stripped of antibody signal by a Western Blot Recycling Kit (Alpha Diagnostic International) and washed with TTBS before reprobing. Complete stripping of signal was verified in preliminary experiments by the absence of signal after treating membranes with ECL and processing as above.

Peroxisomal lactate release. To measure lactate release, isolated peroxisomes (approximately $300 \mathrm{mg}$ ) were incubated at $37^{\circ} \mathrm{C}$ in an incubation medium consisting of: $130 \mathrm{mM} \mathrm{KCl}, 10 \mathrm{mM}$ Hepes, $0.1 \mathrm{mM}$ EGTA, $0.5 \mathrm{mM} \mathrm{NAD}^{+}, 0.1 \mathrm{mM} \mathrm{NADP}^{+}, 1 \mathrm{mM} \mathrm{NH}_{4} \mathrm{Cl}, 2 \mathrm{mg} / \mathrm{ml} \mathrm{BSA}$, $25 \mu \mathrm{g}$ antimycin $\mathrm{A} / \mathrm{ml}$, and $2.5 \mathrm{mM}$ of palmitoyl $\mathrm{CoA}, \mathrm{pH} 7.2$ [4]. To stimulate $\beta$-oxidation pyruvate was added at 1 and $5 \mathrm{mM}$. The resulting lactate release was measured in acid-soluble $\left(\mathrm{HClO}_{4}, 8 \% \mathrm{v} / \mathrm{v}\right)$ supernatants by standard spectrophotometric techniques [13].

Peroxisomal $\beta$-oxidation. After isolation and resuspension in the above incubation medium, but with $1.25 \mathrm{mM}$ palmitoyl-CoA, the oxidation of $\left[1-{ }^{14} \mathrm{C}\right]$ palmitoyl-CoA $(40-60 \mathrm{mCi} / \mathrm{mmol}, \mathrm{NEN})(0.3 \mu \mathrm{Ci}$ added) by peroxisomes (approximately $1-2 \mathrm{mg}$ ) was measured after $20 \mathrm{~min}$ at $37^{\circ} \mathrm{C}$ as $\mathrm{HClO}_{4}(2.5 \% \mathrm{v} / \mathrm{v})$ soluble radioactivity [4]. Total volume of the reaction mixture was $200 \mu \mathrm{l}$ and an equal amount of $\mathrm{HClO}_{4}(2.5 \% \mathrm{v} / \mathrm{v})$ was added after the incubation period. Each tube was centrifuged for $10 \mathrm{~min}$ in a microcentrifuge. Fatty acyl-CoA esters 12 carbons and longer precipitate out of solution leaving products of $\beta$-oxidation as the sole source of radioactivity. Pyruvate $(2 \mathrm{mM})$ was added and then its transport blocked by the addition of $0.5,1$, and $5 \mathrm{mM} \alpha$-cyano-4-hydroxycinnamate (CINN). LDH activity was inhibited by the addition of 10 and $20 \mathrm{mM}$ oxamate $(\mathrm{OX})$. $\beta$-Oxidation was also measured with vehicle (DMSO) alone.
Statistics. Differences in $\beta$-oxidation were analyzed with a one-tail Student's $t$ test after arcsine square root transformation. Significance level was set at $\alpha=0.05$.

\section{Results}

The presence of catalase in peroxisomal fractions was used as a marker for the purity of the isolation procedure (Fig. 2A). There was very little catalase in the $\mathrm{M}$ and $\mathrm{T}$ fractions. The absence of Complex I in the $\mathrm{P}$ and relatively low levels in the $\mathrm{P}+\mathrm{M}$ fractions demonstrates the purity of the preparations and low mitochondrial contamination (Fig. 2B). The presence of both the liver-type monocarboxylate transporter (MCT 2) and MCT 1 were detected in peroxisomal membranes (Figs. $3 \mathrm{~A}$ and $\mathrm{B}$ ). Both of these isoforms were found in all other hepatocyte fractions investigated. Lactate dehydrogenase (LDH)

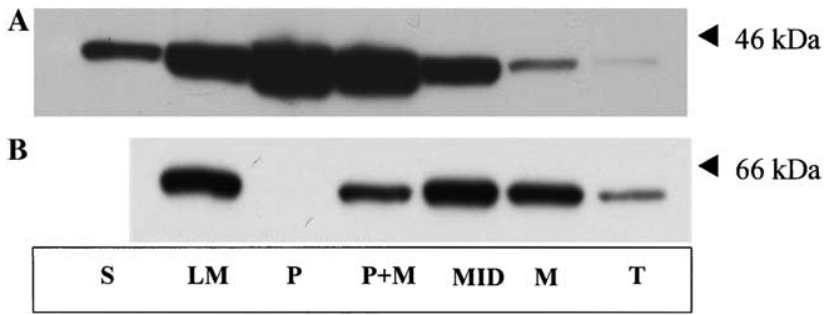

Fig. 2. Purity of peroxisome fractions was verified using Western blots to detect (A) catalase and (B) complex 1 expression in liver homogenate supernatant $(\mathrm{S})$, light mitochondrial (LM), highly purified peroxisomal (P), peroxisomal $(\mathrm{P}+\mathrm{M})$, middle (MID), mitochondrial (M), and lysosomal $(\mathrm{T})$ fractions.

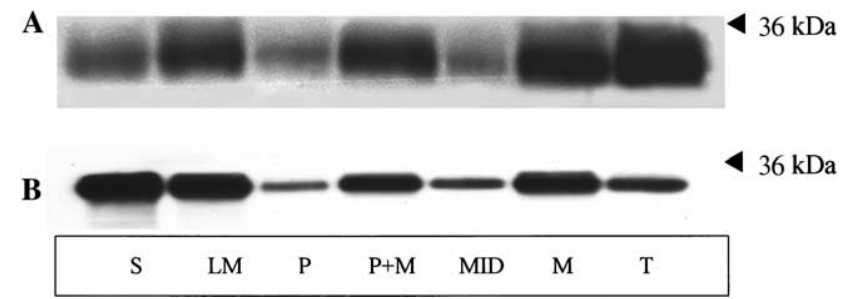

Fig. 3. Western blots for the presence of (A) monocarboxylate transporter 2 (MCT 2) and (B) monocarboxylate transporter 1 (MCT 1) in liver homogenate supernatant (S), light mitochondrial (LM), highly purified peroxisomal $(\mathrm{P})$, peroxisomal $(\mathrm{P}+\mathrm{M})$, middle (MID), mitochondrial (M), and lysosomal (T) fractions.

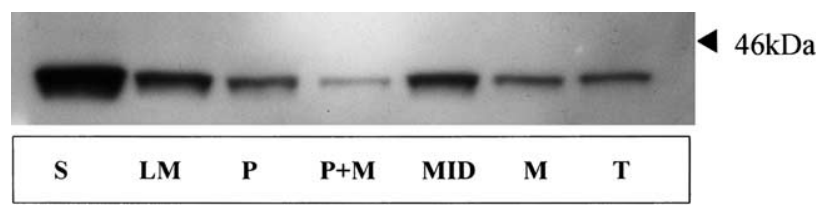

Fig. 4. Western blot for the presence of lactate dehydrogenase (LDH) in liver homogenate supernatant (S), light mitochondrial (LM), highly purified peroxisomal $(\mathrm{P})$, peroxisomal $(\mathrm{P}+\mathrm{M})$, middle (MID), mitochondrial (M), and lysosomal (T) fractions. 


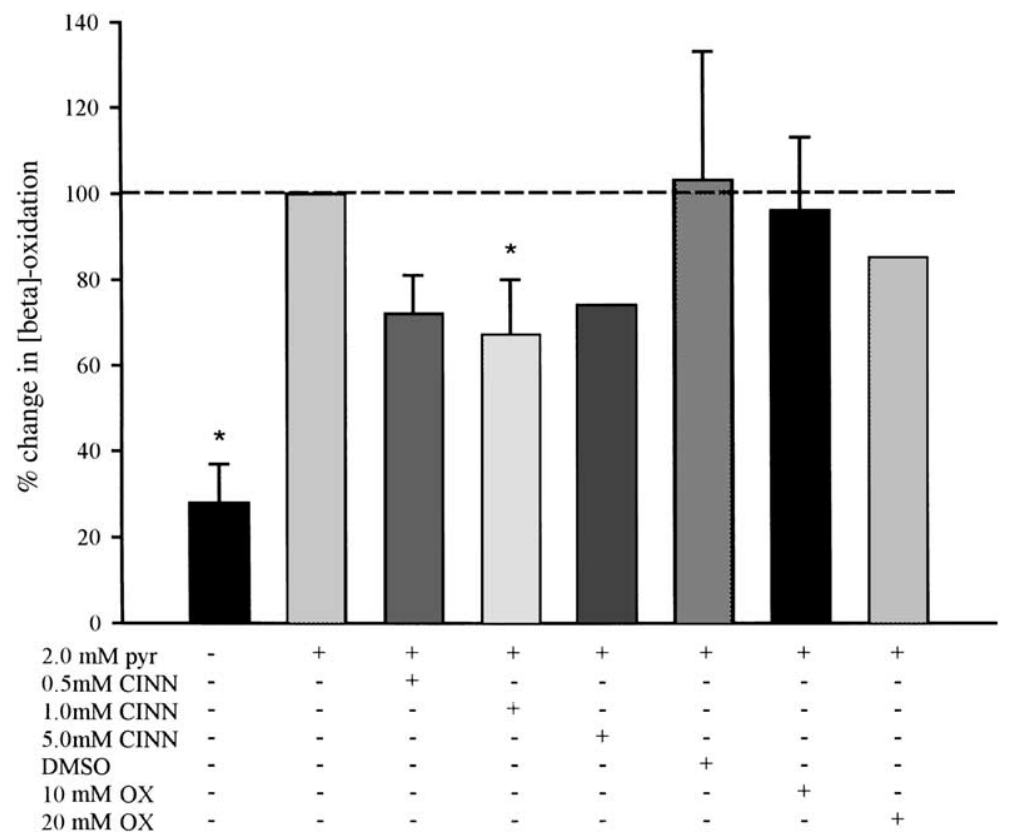

Fig. 5. Percentage change in the $\beta$-oxidation of $\left[1-{ }^{14} \mathrm{C}\right]$ palmitoyl-CoA in isolated peroxisomes from rat liver. Acid soluble radioactivity was measured following the addition of $2 \mathrm{mM}$ pyruvate and in combination with inhibitors of MCT (CINN) or LDH (OX) or vehicle (DMSO). $N=2$ except for $1 \mathrm{mM}$ CINN and $10 \mathrm{mM} \mathrm{OX}, N=3 ; 5 \mathrm{mM} \mathrm{CINN}$ and $20 \mathrm{mM} \mathrm{OX}, N=1$. ${ }^{*}$ Significantly different from pyruvate-stimulated oxidation.

was found in purified peroxisome and mitochondrial fractions (Fig. 4). As well, the LDH isozymic composition of these two fractions tended to be different. Although both organelles were composed primarily of LDH 5 , peroxisomes had relatively more LDH 3 and mitochondria LDH 2 (Fig. 7). Neither fraction had detectable levels of LDH 1. Peroxisomal $\beta$-oxidation, measured as acid soluble radioactivity and expressed relative to pyruvatestimulated rates, was greatly stimulated (4-fold, $p<0.05$ ) by the addition of pyruvate. This stimulation was inhibited $(28-33 \%)$ by the addition of 0.5 and $1(p<0.05)$ and

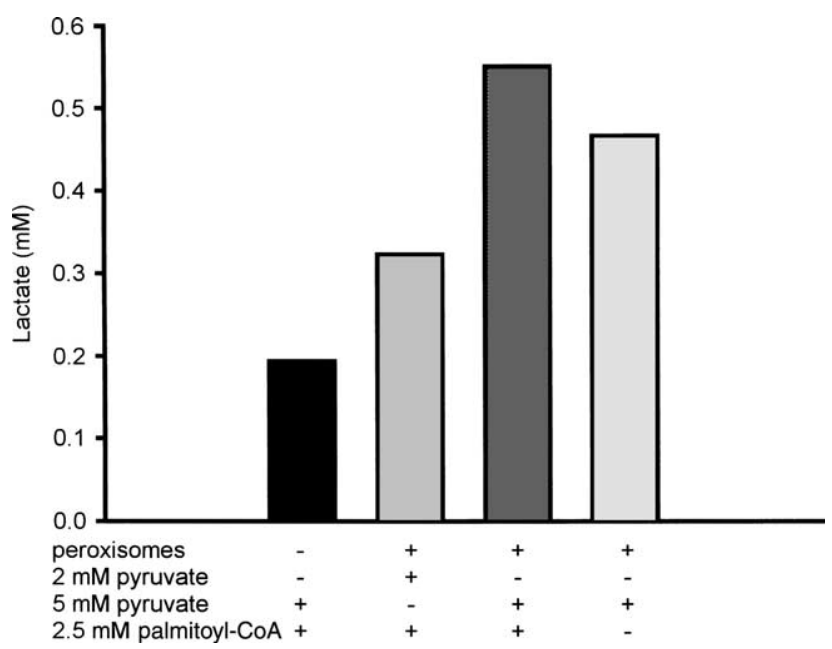

Fig. 6. Lactate released from isolated peroxisome preparations treated with 1 (for $2 \mathrm{~min}$ ) or $5 \mathrm{mM}$ (for $10 \mathrm{~min}$ ) pyruvate in the presence or absence of $2.5 \mathrm{mM}$ palmitoyl-CoA, $N=1$.
$5 \mathrm{mM}$ of the MCT blocker, CINN. Stimulation by pyruvate tended to be inhibited (not statistically different) by the addition of $20 \mathrm{mM} \mathrm{OX} \mathrm{(15 \% ),} \mathrm{an} \mathrm{inhibitor} \mathrm{of} \mathrm{LDH}$ (Fig. 5). The addition of oxaloacetate, another keto-acid involved in redox shuttling, did not significantly enhance the stimulation of $\beta$-oxidation by pyruvate $(N=1$, data not shown). Vehicle alone had no effect on pyruvatestimulated $\beta$-oxidation. Peroxisomes were found to generate lactate upon the addition of 2 and $5 \mathrm{mM}$ pyruvate (Fig. 6). The [lactate] released was diminished by the exclusion of palmitate from the assay medium.

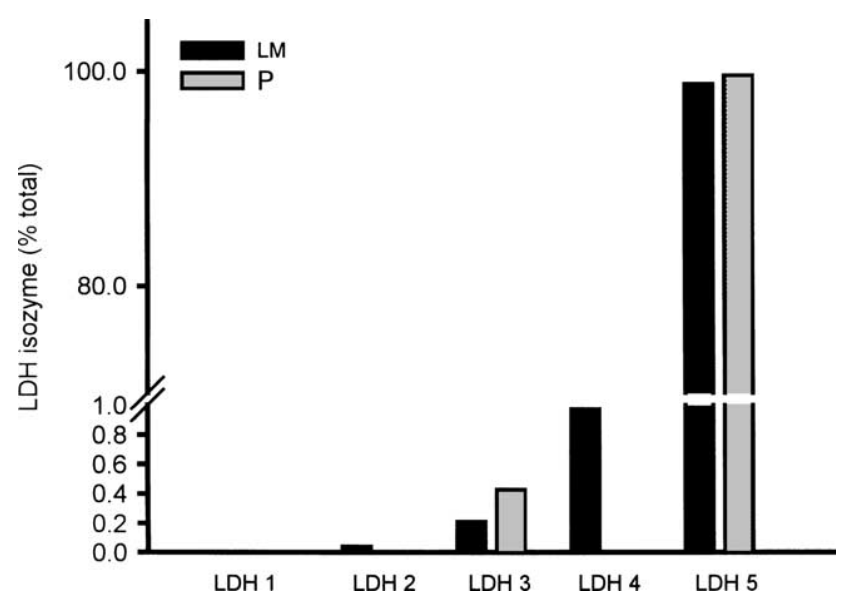

Fig. 7. Lactate dehydrogenase (LDH) isozymes as percent composition in liver homogenate light mitochondria (LM) and highly purified peroxisomes $(\mathrm{P})$ fractions. 


\section{Discussion}

Previously, lactate has been shown as an oxidizable substrate [14-16] and gluconeogenic precursor [17]. Here we demonstrate that because it is more reduced than pyruvate it can also participate in affecting organelle redox balance. As outlined in our model (Fig. 1) pyruvate-lactate exchange across the peroxisome membrane serves to reoxidize NADH essential for the continued functioning of $\beta$-oxidation. This Peroxisomal Lactate Shuttle is a novel mechanism for exchange of these two metabolites and lends support for a similar role in other organelles (i.e., mitochondria) [7]. We have shown that peroxisomal membranes contain MCT 1 and MCT 2 and have confirmed [3] the presence of LDH in this organelle. The functionality of this shuttle was demonstrated by the inhibition of $\beta$-oxidation with blockage of pyruvate entry. Lactate release from intact peroxisomes demonstrates the functionality of both pLDH and membrane MCTs. These data extend our understanding of redox exchanges within cells and the mechanisms involved in peroxisomal $\beta$-oxidation.

MCTs are a family of transporters that transport monocarboxylates across cellular and organelle membranes $[6,18]$. They form an integral part of proposed shuttles that transport lactate between cells (cell-cell lactate shuttle) and within cells (intracellular lactate shuttle or ILS) [19]. One of the putative roles of the ILS is to transfer reducing equivalents from the cytosol into the mitochondrial matrix. Evidence for ILS' role in redox control in mitochondria has been difficult to obtain. Lactate and pyruvate undergo further breakdown once in the mitochondria matrix making it difficult to tease out their respective roles in the shuttling of reducing equivalents to the electron transport chain. Peroxisomes, on the other hand, lack the enzymes to catabolize monocarboxylates. Although they are known to contain LDH [3], confirmed in this study (Fig. 4), they are not known to contain any other glycolytic or any tricarboxylic acid cycle enzymes. This provides an ideal system in which to test the hypothesis that lactate-pyruvate shuttling can affect redox in an organelle. We show evidence that peroxisomal membranes contain MCTs (Fig. 3). These MCTs serve to transport pyruvate from the cytosol into peroxisomes and to export lactate back to the cytosol (see model in Fig. 1). How these MCTs end up in the peroxisomal membrane or how the density is controlled when an animal is exposed to stressors is unknown. To date there has been only one promoter analysis study of MCT 1 [20] and nothing is known about the induction of MCT expression to stimuli known to increase peroxisomal density.

An earlier study suggested a link between $\mathrm{pLDH}$ and the reoxidation of NADH generated by the $\beta$-oxidation of palmitoyl-CoA [3]. We have shown here that this occurs by a lactate-pyruvate shuttle that is dependent on the transport of these monocarboxylates into peroxisomes by MCT 1 and MCT 2. As outlined in our model (Fig. 1) MCTs and pLDH participate in a pyruvate-lactate shuttle in peroxisomes. The shuttle is essential to maintaining proper redox inside the peroxisomes to ensure that $\beta$-oxidation can continue. It is possible that lactate is the keto-acid transported into peroxisomes for the regeneration of $\mathrm{NAD}^{+}$in the cytosol. Reoxidation of NADH in the cytosol by cLDH would generate lactate that could be oxidized to pyruvate in the peroxisome, generating $\mathrm{H}_{2} \mathrm{O}_{2}$ as a product. This seeming wasteful process would not generate any ATP but would serve to balance cytosol redox when mitochondrial oxidation of NADH cannot keep pace with the needs of the cell [21]. This scenario seems counterproductive since it would also serve to inhibit $\beta$ oxidation in the peroxisome and seems unlikely to occur under normal circumstances.

We have now found that LDH exists in at least three distinct pools in the cell. It occurs in the cytosol as the terminal enzyme in anaerobic glycolysis, in mitochondria to convert lactate to pyruvate in the inner membrane space, and now in peroxisomes as part of a lactate-pyruvate shuttle. In all three cases the role of LDH is in maintaining proper redox balance in the compartments in which it exists. Isozymic differences between the three cellular compartments [7,22] support the purity of our cellular fractionation process. It is still unknown as to how these proteins are targeted to their specific location, but there is evidence that the pools respond differently to perturbations, e.g., hypoxia [22]. We suggest that there must be specific targeting mechanisms for the incorporation of not only LDH but also MCTs into different organelles.

\section{Conclusion}

Peroxisomes represent an ideal system in which to assess the function of lactate-pyruvate shuttling in organelle redox balance. Since this system involves neither the consumption nor production of lactate or pyruvate, their exchange represents a true substrate cycle. Together with recent data showing the effect of pyruvate on peroxisomal $\beta$-oxidation [3], this study demonstrates that peroxisomes contain MCTs and $\mathrm{pLDH}$ that transport pyruvate and convert it to lactate affecting the rate of $\beta$-oxidation.

\section{Acknowledgments}

This research was supported by National Institutes of Health Grants DK-19577 and AR-42906 to G.A.B. G.B.M. was a recipient of a Natural Sciences and Engineering Research Council of Canada Postdoctoral Fellowship and a Heart and Stroke Scientific Research Corporation of Canada (HSSRCC) Fellowship. 


\section{References}

[1] P.B. Lazarow, C. De Duve, A fatty acyl-CoA oxidizing system in rat liver peroxisomes; enhancement by clofibrate, a hypolipidemic drug, Proc. Natl. Acad. Sci. USA 73 (1976) 2043-2046.

[2] C.W. van Roermund, Y. Elgersma, N. Singh, R.J. Wanders, H.F. Tabak, The membrane of peroxisomes in Saccharomyces cerevisiae is impermeable to $\mathrm{NAD}(\mathrm{H})$ and acetyl-CoA under in vivo conditions, EMBO J. 14 (1995) 3480-3486.

[3] E. Baumgart, H.D. Fahimi, A. Stich, A. Volk1, L-Lactate dehydrogenase A4- and A3B isoforms are bona fide peroxisomal enzymes in rat liver, J. Biol. Chem. 271 (1996) 3846-3855.

[4] H. Osmundsen, Factors which can influence $\beta$-oxidation by peroxisomes isolated from livers of clofibrate treated rats. Some properties of peroxisomal fractions isolated in a self generated Percoll gradient by vertical rotor centrifugation, Int. J. Biochem. 14 (1982) 905-914.

[5] D.A. Roth, G.A. Brooks, Lactate transport is mediated by a membrane-bound carrier in rat skeletal muscle sarcolemmal vesicles, Arch. Biochem. Biophys. 279 (1990) 377-385.

[6] A.P. Halestrap, N.T. Price, The proton-linked monocarboxylate transporter (MCT) family: structure, function and regulation, Biochem. J. 343 (1999) 281-299.

[7] G.A. Brooks, H. Dubouchaud, M. Brown, J.P. Sicurello, C.E. Butz, Role of mitochondrial lactate dehydrogenase and lactate oxidation in the intracellular lactate shuttle, Proc. Natl. Acad. Sci. USA 96 (1999) 1129-1134.

[8] D. Valanti, L. De Bari, A. Atlante, S. Passarella, L-Lactate transport into rat heart mitochondria and reconstruction of the $\mathrm{L}-$ lactate/pyruvate shuttle, Biochem. J. 364 (2002) 101-104.

[9] F.G. Gallina, N.M. Gerez de Burgos, C. Burgos, C.E. Coronel, A. Blanco, The lactate/pyruvate shuttle in spermatozoa: operation in vitro, Arch. Biochem. Biophys. 308 (1994) 515-519.

[10] J. Calvin, P.K. Tubbs, Mitochondrial transport processes and oxidation of NADH by isotonically-treated boar spermatozoa, Eur. J. Biochem. 89 (1978) 315-320.
[11] P.P. Van Veldhoven, E. Baumgart, G.P. Mannaerts, Iodixanol (Optiprep), an improved density gradient medium for the isoosmotic isolation of rat liver peroxisomes, Anal. Chem. 237 (1996) $17-23$.

[12] R.C. Poole, C.E. Sansom, A.P. Halestrap, Studies of the membrane topology of the rat erythrocyte $\mathrm{H}^{+}$/lactate cotransporter (MCT1), Biochem. J. 320 (1996) 817-824.

[13] H.U. Bergmeyer, Methods of Enzymatic Analysis, vol. 3, Academic Press, New York, 1974.

[14] C.M. Donovan, G.A. Brooks, Endurance training affects lactate clearance, not lactate production, Am. J. Physiol. 244 (1983) E83E92.

[15] W.C. Stanley, E.W. Gertz, J.A. Wisneski, D.L. Morris, R.A. Neese, G.A. Brooks, Systemic lactate kinetics during graded exercise in man, Am. J. Physiol. 249 (1985) E595-E602.

[16] B.C. Bergman, E.E. Wolfel, G.E. Butterfield, G.D. Lopaschuk, G.A. Casazza, M.A. Horning, G.A. Brooks, Active muscle and whole body lactate kinetics after endurance training in men, $\mathrm{J}$. Appl. Physiol. 87 (1999) 1684-1696.

[17] G.A. Brooks, C.M. Donovan, Effect of endurance training on glucose kinetics during exercise, Am. J. Physiol. 244 (1983) E505E512.

[18] G.A. Brooks, M.A. Brown, C.E. Butz, J.P. Sicurello, H. Dubouchaud, Cardiac and skeletal muscle mitochondria have a monocarboxylate transporter MCT1, J. Appl. Physiol. 87 (1999) 17131718.

[19] G.A. Brooks, Intra- and extra-cellular lactate shuttles, Med. Sci. Sports Exerc. 32 (2000) 790-799.

[20] M.A. Cuff, S.P. Shirazi-Beechey, The human monocarboxylates transporter, MCT1: genomic organization and promoter analysis, Biochem. Biophys. Res. Commun. 292 (2002) 1048-1056.

[21] W.M. Becker, The World of the Cell, Benjamin/Cummings, Menlo Park, CA, 1986.

[22] G.B. McClelland, G.A. Brooks, Changes in MCT1, MCT4 and LDH expression are tissue specific in rats after long-term hypobaric hypoxia, J. Appl. Physiol. 92 (2002) 1573-1584. 